\title{
SResearch Square

\section{Effects of a community physical activity program on the number of antihypertensive drugs used by elderly women with arterial hypertension: a cross- sectional study}

Claudio Andre Barbosa de Lira ( $\square$ andre.claudio@gmail.com ) https://orcid.org/0000-0001-5749-6877

\section{Rafaela Gomes Santos}

Universidade do Estado da Bahia

Ariádny Brandão Gomes

Universidade Federal do Espirito Santo Radio Universitaria 104.7 FM

Douglas Assis Teles Santos

Universidade do Estado da Bahia https://orcid.org/0000-0002-7664-5468

Marília Santos Andrade

Universidade Federal de Sao Paulo

Rodrigo Luiz Vancini

Universidade Federal do Espirito Santo

Research article

Keywords: exercise, blood pressure, drug therapy

Posted Date: July 8th, 2019

DOI: https://doi.org/10.21203/rs.2.11069/v1

License: (c) (i) This work is licensed under a Creative Commons Attribution 4.0 International License.

Read Full License 


\section{Abstract}

Background Physical exercise is an important tool to manage systemic arterial hypertension. However, less is known about the effect of physical exercise on the number of antihypertensive drugs used by older adults. Objectives The aim was to compare the number of antihypertensive drugs used by older female adults with a low level of physical activity with the number used by those with a high level of physical activity, and to verify how many participants used more than two antihypertensive drugs. Methods Twenty-eight physically active older women with systemic arterial hypertension who participated in a physical activity program for community-dwelling older female adults were divided into two groups: participants that presented lower habitual physical activity levels were placed in group 1 and participants that presented higher habitual physical activity levels were placed in group 2, according to the Baecke questionnaire. In addition, the number of antihypertensive drugs used by participants was collected. Results The number of ingested antihypertensive tablets was 2.0 (median) for both groups investigated. There was no significant difference between groups regarding the number of antihypertensive tablets ingested $(p>0.05)$. Although there was no statistical difference, a higher proportion of participants from the lower physical activity group used more than two antihypertensive drugs. Conclusions The level of habitual physical activity did not affect the number of antihypertensive tablets used by hypertensive women.

\section{Introduction}

Aging of the population and the increased prevalence of chronic noncommunicable diseases among older adults are major challenges facing society and the medical community [1]. Among these diseases, systemic arterial hypertension is a significant cause and consequence of disability among older adults [2, 3].

Systemic arterial hypertension is a multifactorial clinical condition characterized by elevated and sustained blood pressure levels. It is often associated with functional and/or structural changes in target organs (e.g. heart, brain, kidneys and blood vessels) and metabolic changes, with a consequent increase in the risk of cardiovascular events $[3,4]$.

According to the High Blood Pressure Clinical Practice Guideline, $46 \%$ of the population has systemic arterial hypertension. In addition, the prevalence increases dramatically with increasing age and is higher in black people than in Caucasians, Asians and Hispanics [5]. Furthermore, the prevalence of systemic arterial hypertension is lower in women than in men up to the fifth decade, but it is higher at the end of life [5]. For these reasons, the study of systemic arterial hypertension in older women is an area of growing interest.

The most frequently used treatment for systemic arterial hypertension is antihypertensive medication [6, 7]. However, in the last decades alternative therapies such as physical exercise have been gaining notoriety for the important beneficial effects on blood pressure of patients with systemic arterial 
hypertension [8-10]. Indeed, a plethora of studies showed that physical exercise may be a potential nonpharmacological treatment for the improvement of blood pressure in patients with hypertension [11]. Because of these effects, some authors have suggested that exercise may reduce or even replace pharmacological treatment. In this sense, Maruf et al. (2016) investigated the effects of aerobic exercise combined with antihypertensive drugs on blood pressure and the number of antihypertensive drugs used by individuals with hypertension. The authors found that the combination of aerobic exercise and antihypertensive drugs reduces the number of antihypertensive drugs needed to achieve blood pressure control and enhances blood pressure control in individuals with hypertension on two antihypertensive drugs. In another study, Dimeo et al. (2012) showed that physical exercise is able to decrease blood pressure even in participants with low responsiveness to medical treatment, and concluded that physical exercise should be included in the therapeutic approach to resistant hypertension. Reid, Maher, and Jennings (2000), showed that $71 \%$ of patients submitted to lifestyle management (including physical exercise) who had been well controlled on antihypertensive medication were able to remain normotensive and free of therapy for 9 months.

From the above, the role of exercise in the control of blood pressure in individuals with systemic arterial hypertension is indubitable. For this reason, there are many governmental initiatives that aim to encourage the participation of the population in physical activity programs. These initiatives are community physical activity programs characterized by outdoor activities that take place in public spaces, such as squares, beaches and parks. In Brazil, the main concern about these community physical activity programs is the lack of individualization of exercise prescription, in some cases lack of specialized professionals (coaches and/or physical education professionals) to guide and supervise participants, and adequate exercise facilities. Thus, it is reasonable to assume that factors relating to physical training, such as intensity, weekly frequency and volume, are not strictly controlled. As a consequence, the health benefits may not be gained [15]. Specifically, in the case of patients with systemic arterial hypertension, it is expected, in addition to a decrease in blood pressure, a decrease in the amount of antihypertensive drugs used [7, 12].

Given that a myriad of studies showed beneficial effects of physical exercise on the blood pressure of individuals with systemic arterial hypertension, physical exercise may complement the blood pressurelowering effects of antihypertensive drugs $[12,16]$, thereby reducing the number and/or dose of drugs required $[7,12]$. This matter is very important because a number of daily antihypertensive drugs tablets intake greater than two has been associated with no adherence to drug therapy [17]. Considering that drugs cost money and may produce negative side effects (Kaplan 1998), it is very important to investigate strategies that will lead to a reduction in the daily antihypertensive drugs tablets intake. Therefore, the aim of the present study was to compare the number of antihypertensive drugs taken by women who have a low level of physical activity with the number of antihypertensive drugs taken by those with a high level of activity. We also wanted to establish how many participants in one group took more than two antihypertensive drugs compared with those participants from the other group. We hypothesized that the higher the habitual physical activity levels the lower the number of antihypertensive drugs used and that these participants would use two or less antihypertensive drugs. It is noteworthy that 
investigating community physical activity programs has great ecological validity because they reflect real situations and scenarios.

\section{Materials And Methods Participants}

A cross-sectional study was performed comprising 28 female older participants (a convenience sample out of a total of 280 people) who were recruited using advertisements placed in a physical activity program for community-dwelling older adults (located in the city of Serra, Espírito Santo, Brazil) and consisted of women that used antihypertensive drugs. The inclusion criteria used were: being physically active (participated in a physical activity program for community-dwelling older adults); to use regularly at least one antihypertensive drug prescribed by physicians; and aged $\geq 60$ years. Sedentary and male older adults were excluded from the sample. After a clear explanation of the experimental procedures, including the risks and benefits of participation, written consent was obtained. The age and anthropometrical characteristics of the participants are presented in Table 1. Ethics approval for all experimental procedures was granted by the Federal University of Espírito Santo Human Research Ethics Committee and conformed to the principles outlined in the Declaration of Helsinki.

Table 1 near here

\section{Study design}

Participants answered a questionnaire about the use of antihypertensive drugs and habitual physical activity levels using the Baecke Habitual Physical Activity Questionnaire [18]. Because of the low educational level of the sample, an interview was used to fill out the questions. The participants were divided into two groups according to the questionnaire results. To this end, we used the median values of total score for habitual physical activity provided by the Baecke questionnaire. Thus, participants who presented lower habitual physical activity levels (values below median) were placed in group 1 and those that presented higher (values above median) were placed in group 2.

\section{The physical activity program for community- dwelling older adults}

The physical activity program for community-dwelling older adults (named Programa de Orientação ao Exercício Físico) provides health-promotion actions by encouraging physical activity and healthy eating, with a view to disease prevention. Group classes are offered, 2-3 times per week, with an orientation to aerobic exercise (running and walking), localized gymnastics and stretching, among others. Each class lasted 45-50 minutes. 


\section{Antihypertensive drugs use questionnaire}

The questionnaire comprised questions intended to capture information about the number of antihypertensive drugs used. It is worth noting that the number of antihypertensive drugs was counted regardless of whether it was a full or half-dose.

\section{Baecke Habitual Physical Activity Questionnaire}

The habitual level of physical activity was evaluated by the Baecke questionnaire [18]. This questionnaire consists of 16 questions involving three habitual physical activity scores relating to the previous 12 months: occupational physical activity (eight questions); physical exercise in leisure time (four questions); and leisure and locomotion activities (four questions). As the participants were retired or housewives, for the purposes of the present study we evaluated only the physical exercise in leisure time and leisure and locomotion activity domains. The total score for habitual physical activity was obtained by summing the physical exercise in leisure and leisure and locomotion activity scores. The higher the score achieved, the higher the level of habitual physical activity.

\section{Statistical analyses}

As the habitual physical activity level and number of antihypertensive drugs used by participants were not normally distributed according to the Shapiro-Wilk normality test, the data were expressed as medians (Quartile 1 Quartile 3) and the Mann-Whitney test was used to compare variables between the groups [group 1 (lower habitual physical activity) vs. group 2 (higher habitual physical activity)]. Fisher's exact test was used to check for an association between the level of habitual physical activity (group 1 and group 2) and the number of antihypertensive drugs consumed ( $\leq 2$ and $>2$ ). Cramer's $\vee$ was used to express effect size in these analyses in order to test the strength for any association or practical significance from the Fisher's exact test analysis. It could be interpreted as follows: effect size of 0.1 is small, effect size of 0.3 is medium, and an effect size of 0.5 is large (Cohen 1988). The SPSS statistical package, version 23.0 (IBM Corp., Armonk, NY) was used for statistical analysis, and a significance level of 0.05 was set for all statistical tests.

\section{Results}

Antihypertensive medication used by participants were diuretics $(n=13)$, calcium-channel blockers $(n=6)$, $\beta$-blockers ( $n=11)$, angiotensin-converting enzyme inhibitors $(n=5)$, angiotensin II type 1 blockers $(n=11)$ and $\mathrm{a}-2$ adrenergic agonists $(\mathrm{n}=2)$. The sum of the number of antihypertensive drugs does not result in the total number of participants since there were participants who used more than one drug. 
Table 2 shows the habitual level of physical activity evaluated by the Baecke questionnaire according to the median [lower (group 1) vs. higher (group 2) habitual physical activity levels] of the questionnaire score. As expected, the Mann-Whitney test revealed significant differences between groups for subscales and total score.

Table 2 near here

Table 3 shows the participants' consumption of antihypertensive drugs [lower (group 1) vs. higher (group 2) habitual physical activity levels]. The Mann-Whitney test revealed no significant differences.

Table 3 near here

Table 4 shows the association of participants who used more than two antihypertensive drugs between groups. The Fisher's exact test revealed no significant association between variables. However, the effect size expressed by Cramer's $V$ was 0.316 , indicating that there is a medium to large effect.

Table 4 near here

\section{Results}

Antihypertensive medication used by participants were diuretics $(n=13)$, calcium-channel blockers $(n=6)$, $\beta$-blockers ( $n=11)$, angiotensin-converting enzyme inhibitors $(n=5)$, angiotensin II type 1 blockers $(n=11)$ and $a-2$ adrenergic agonists $(n=2)$. The sum of the number of antihypertensive drugs does not result in the total number of participants since there were participants who used more than one drug.

Table 2 shows the habitual level of physical activity evaluated by the Baecke questionnaire according to the median [lower (group 1) vs. higher (group 2) habitual physical activity levels] of the questionnaire score. As expected, the Mann-Whitney test revealed significant differences between groups for subscales and total score.

Table 2 near here

Table 3 shows the participants' consumption of antihypertensive drugs [lower (group 1) vs. higher (group 2) habitual physical activity levels]. The Mann-Whitney test revealed no significant differences.

Table 3 near here

Table 4 shows the association of participants who used more than two antihypertensive drugs between groups. The Fisher's exact test revealed no significant association between variables. However, the effect size expressed by Cramer's $\vee$ was 0.316 , indicating that there is a medium to large effect.

Table 4 near here

\section{Discussion}


The main aim of the current study was to verify whether the number of antihypertensive drugs intake is different between a sample of two groups of female older adults with different habitual physical activity levels (lower vs. higher). We found that there were no significant differences in the number of antihypertensive drugs consumed in a sample categorized according to habitual physical activity level provided by the Baecke questionnaire. However, we found that a higher proportion of participants from Group 2 take two or less antihypertensive tablets.

Previously, several studies have been conducted to assess the effectiveness of physical exercise to manage systemic arterial hypertension [19,20]. Overall, these studies showed the beneficial effects of physical exercise by using protocols that are controlled, standardized and structured. The existing exercise recommendations for hypertension advocate that with regards to frequency, aerobic exercise should be undertaken on most (preferably all) days of the week and resistance exercise on 2 to 3 days in that same week [20]. With regards to intensity, the recommendation is for moderate intensity aerobic exercise (i.e., 40 to $<60 \%$ of maximal oxygen uptake or heart rate reserve; $11-13$ rating of perceived exertion on the $6-20$ Borg Scale and moderate intensity dynamic resistance exercise $(60 \%$ to $80 \%$ one repetition maximum) [20]. In terms of time, the duration of exercise should total 150 min or more per week [20]. Finally, progression should be gradual, avoiding large increases in any exercise components (frequency, intensity and time).

From the above, the lack of difference in the number of antihypertensive drugs intake between groups (lower vs. higher habitual physical activity level) found in the current study could probably be attributed to characteristics of the physical activity program for community-dwelling older adults. Briefly, this program is characterized by a lack of standardization with regards to exercise intensity, session duration and weekly frequency. According to Dimeo et al. (2012), in daily life, the performance of exercise in arterial hypertension is usually not monitored by a sophisticated method such as lactate concentration. Therefore, the recommendation of training intensity has to be kept more practical. Therefore, it is reasonable to assume that physical exercise performed by the participants did not follow the guidelines of exercise prescription for managing systemic arterial hypertension. However, the older women that we studied are part of a public health program that seeks to promote and educate for health and quality of life and to change negative health habits, which in our view is a strong point of the program.

Another aspect that can be considered to explain the results obtained by the current study is that -in Brazil, at least - physicians are not involved with concepts related to exercise and sports science. According to the Centers for Disease Control and Prevention [CDC] (1998), only $19 \%$ of physicians counsel patients about physical activity. Probably, these results can be attributed because physicians are not interested in learning about exercise science due to time pressures. As a result, it is reasonable to assume that physicians could attribute the clinical improvements of a patient to pharmacological treatment rather than physical exercise engagement, and for this reason did not decrease the prescribed number of drugs. Corroborating this assertion, it has already been demonstrated that only a few patients receive exercise counseling from physicians for managing blood pressure to improve health outcomes [22]. 
A daily administration of more than two antihypertensive drugs has been associated with nonadherence to drug therapy [17]. Individuals with systemic arterial hypertension who either discontinue medication or are nonadherent to the prescribed drug therapy are at risk of developing cardiovascular complications [23]. In the current study, 28.6\% (six participants in group 1 and two in group 2) used more than two antihypertensive drugs. Although there was no significant association of participants who used more than two antihypertensives drugs between groups, the number of participants in group 2 (higher levels of habitual physical activity) who ingested more than two drugs was one third of that of group 1 (lower levels of habitual physical activity), consequently the proportion of participants who used two or less antihypertensive tablets is higher in Group 2. The effect size of this analysis expressed by Cramer's $V$ was medium to large. This result has clinical significance, because it highlights that although the physical activity program for community-dwelling older adults did not strictly follow current recommendations for exercise to reduce arterial hypertension, Group 2 showed an important effect, since the number of participants who used more than two antihypertensive drugs was lower; this is probably because the intensity and volume of exercise performed by Group 2 were higher. Furthermore, this result suggests that the need to prescribe a third antihypertensive drug will be minimized in these individuals, which suggests that exercise in conjunction with antihypertensive drug therapy improves blood pressure control and may even lead to fewer antihypertensive drugs prescribed. Finally, primary health-care professionals, aware of these results, could recommend that patients with systemic arterial hypertension increase the amount of physical activity in order to decrease the number of antihypertension drugs ingested.

\section{Declarations}

\section{Acknowledgements}

The authors would like to thank Mr. Claudino Rodrigues dos Santos Júnior and Mr. Marcelo dos Santos Teixeira (Programa de Orientação ao Exercício Físico, Serra, Espírito Santo) for their technical support, and all the participants who volunteered their time to participate in the study. CABL had a fellowship from the Coordenação de Aperfeiçoamento de Pessoal de Ensino Superior, Programa Nacional de PósDoutorado (CAPES/PNPD - Coordination for the Improvement of Higher Education Personnel, National Postdoctoral Program, Brazil). RLV is a productivity fellow at the Fundação de Amparo à Pesquisa e Inovação do Espírito Santo (FAPES) agency (Edital No 18/2018 - Bolsa Pesquisador Capixaba).

Ethics, consent and permissions: All subjects were informed of the intent and experimental procedures and written informed consent was obtained from each participant before any data were collected. All procedures involved in this study were approved by the Federal University of Espírito Santo Ethics Committee (protocol number [CAAE]: 63228215.1.3001.5505) and followed the principles outlined in the Declaration of Helsinki.

Consent for publication: Not applicable

Competing interests: The authors declare that they have no competing interests. 
Funding: This work was supported by the Fundação de Amparo à Pesquisa e Inovação do Espírito Santo (FAPES, Edital nº. 007/2014 - Universal - Projeto Integrado de Pesquisa; $n^{\circ}$. FAPES: 0423/2015 - grant number: 67640427), Fundação de Amparo à Pesquisa e Inovação do Espírito Santo (FAPES)/Conselho Nacional de Desenvolvimento Científico e Tecnológico (CNPq) (edital FAPES/CNPq/Decit-SCTIEMS/SESA nº 03/2018 - programa de pesquisa para o SUS - PPSUS; grant number: 212/2018). Brazil.

\section{References}

1. WHO. Ageing and health. World Health Organization . 2015. https://www.who.int/en/news-room/factsheets/detail/ageing-and-health. Accessed 19 Dec 2018.

2. Sun Z. Aging, Arterial Stiffness, and Hypertension. Hypertension. 2015;65:252-6. doi:10.1161/HYPERTENSIONAHA.114.03617.

3. World Health Organization. A global brief on Hypertension - World Health Day 2013. World Health Organization. 2013;:1-40.

4. Cushman WC. The burden of uncontrolled hypertension: morbidity and mortality associated with disease progression. J Clin Hypertens. 2003;5 3 Suppl 2:14-22.

http://www.ncbi.nlm.nih.gov/pubmed/12826766. Accessed 19 Dec 2018.

5. Whelton PK, Carey RM, Aronow WS, Casey DE, Collins KJ, Dennison Himmelfarb C, et al. 2017 ACC/AHA/AAPA/ABC/ACPM/AGS/APhA/ASH/ASPC/NMA/PCNA Guideline for the Prevention, Detection, Evaluation, and Management of High Blood Pressure in Adults: Executive Summary: A Report of the American College of Cardiology/American Heart Association Task Force on Clinical Practice Guidelines. Hypertension. 2018;71:1269-324. doi:10.1161/HYP.0000000000000066.

6. Cardoso Jr CG, Gomides RS, Queiroz ACC, Pinto LG, Lobo F da S, Tinucci T, et al. Acute and chronic effects of aerobic and resistance exercise on ambulatory blood pressure. Clinics. 2010;65:317-25. doi:10.1590/S1807-59322010000300013.

7. Miller ER, Erlinger TP, Young DR, Jehn M, Charleston J, Rhodes D, et al. Results of the Diet, Exercise, and Weight Loss Intervention Trial (DEW-IT). Hypertension. 2002;40:612-8. http://www.ncbi.nlm.nih.gov/pubmed/12411452. Accessed 19 Dec 2018.

8. Belozo FL, Katashima CK, Cordeiro A V, Lenhare L, Alves JF, Silva VRR. Effects of ninety minutes per week of continuous aerobic exercise on blood pressure in hypertensive obese humans. $J$ Exerc Rehabil. 2018;14:126-32. doi:10.12965/jer.1835162.581.

9. Mancia G, Fagard R, Narkiewicz K, Redón J, Zanchetti A, Böhm M, et al. 2013 ESH/ESC Guidelines for the management of arterial hypertension. J Hypertens. 2013;31:1281-357.

doi:10.1097/01.hjh.0000431740.32696.cc. 
10. Pescatello LS, Franklin BA, Fagard R, Farquhar WB, Kelley GA, Ray CA, et al. American College of Sports Medicine position stand. Exercise and hypertension. Med Sci Sports Exerc. 2004;36:533-53. http://www.ncbi.nlm.nih.gov/pubmed/15076798. Accessed 19 Dec 2018.

11. Wen $\mathrm{H}$, Wang L. Reducing effect of aerobic exercise on blood pressure of essential hypertensive patients. Medicine (Baltimore). 2017;96:e6150. doi:10.1097/MD.0000000000006150.

12. Maruf FA, Akinpelu AO, Salako BL, Akinyemi JO. Effects of aerobic dance training on blood pressure in individuals with uncontrolled hypertension on two antihypertensive drugs: a randomized clinical trial. J Am Soc Hypertens. 2016;10:336-45. doi:10.1016/j.jash.2016.02.002.

13. Dimeo F, Pagonas N, Seibert F, Arndt R, Zidek W, Westhoff TH. Aerobic Exercise Reduces Blood Pressure in Resistant Hypertension. Hypertension. 2012;60:653-8.

doi:10.1161/HYPERTENSIONAHA.112.197780.

14. Reid CM, Maher T, Jennings GL, Heart Project Steering Committee. Substituting lifestyle management for pharmacological control of blood pressure: a pilot study in Australian general practice. Blood Press. 2000;9:267-74. http://www.ncbi.nlm.nih.gov/pubmed/11193130. Accessed 19 Dec 2018.

15. Heath GW, Parra DC, Sarmiento OL, Andersen LB, Owen N, Goenka S, et al. Evidence-based intervention in physical activity: lessons from around the world. Lancet. 2012;380:272-81. doi:10.1016/S0140-6736(12)60816-2.

16. Maruf F, Akinpelu A, Salako B. Effects of aerobic exercise and drug therapy on blood pressure and antihypertensive drugs: a randomized controlled trial. Afr Health Sci. 2013;13:1-9. doi:10.4314/ahs.v13i1.1.

17. Familoni BO, Ogun SA, Aina AO. Knowledge and awareness of hypertension among patients with systemic hypertension. J Natl Med Assoc. 2004;96:620-4. http://www.ncbi.nlm.nih.gov/pubmed/15160976. Accessed 20 Dec 2018.

18. Baecke JAH, Burema J, Frijters JE. A short questionnaire for the measurement habitual physical activity in epidemiological. Am J Clin Nutr. 1982;:936-42.

19. Cornelissen VA, Fagard RH. Effects of Endurance Training on Blood Pressure, Blood PressureRegulating Mechanisms, and Cardiovascular Risk Factors. Hypertension. 2005;46:667-75. doi:10.1161/01.HYP.0000184225.05629.51.

20. Pescatello LS, MacDonald H V, Lamberti L, Johnson BT. Exercise for Hypertension: A Prescription Update Integrating Existing Recommendations with Emerging Research. Curr Hypertens Rep. 2015;17:87. doi:10.1007/s11906-015-0600-y.

21. Centers for Disease Control and Prevention (CDC). Missed opportunities in preventive counseling for cardiovascular disease-United States, 1995. MMWR Morb Mortal Wkly Rep. 1998;47:91-5. 
http://www.ncbi.nlm.nih.gov/pubmed/9480410. Accessed 20 Dec 2018.

22. Halm J, Amoako E. Physical activity recommendation for hypertension management: does healthcare provider advice make a difference? Ethn Dis. 2008;18:278-82.

http://www.ncbi.nlm.nih.gov/pubmed/18785439. Accessed 20 Dec 2018.

23. Kaplan NM. Treatment of hypertension: insights from the JNC-VI report. Am Fam Physician. 1998;58:1323-30. http://www.ncbi.nlm.nih.gov/pubmed/9803197. Accessed 20 Dec 2018.

\section{Tables}

Table 1: General participant characteristics

\begin{tabular}{lcc}
\hline & Mean \pm SD & Min-Max \\
\hline Age (years) & $69.5 \pm 6.4$ & $60.0-85.0$ \\
Height $(\mathrm{cm})$ & $1.60 \pm 0.08$ & $1.50-1.80$ \\
Body mass $(\mathrm{kg})$ & $67.4 \pm 10.2$ & $53.0-91.0$ \\
BMI $\left(\mathrm{kg} . \mathrm{m}^{-2}\right)$ & $27.0 \pm 2.8$ & $21.9-31.3$ \\
\hline
\end{tabular}

SD: standard deviation. Min-Max: minimum and maximum values. BMI: body mass index.

Table 2. Baecke questionnaire subscales and total score

\begin{tabular}{lccc}
\hline Baecke's results & Group 1 (n=14) & Group 2 (n=14) & $P$ \\
\hline Physical exercise in leisure time & $2.8[2.5-2.8]$ & $3.3[3.0-3.6]$ & 0.0001 \\
Leisure and locomotion activity & $2.5[2.5-3.0]$ & $3.5[3.3-3.6]$ & 0.0001 \\
\hline Total score $^{\mathrm{a}}$ & $5.6[5.2-5.8]$ & $6.5[6.2-7.1]$ & 0.0001 \\
\hline
\end{tabular}

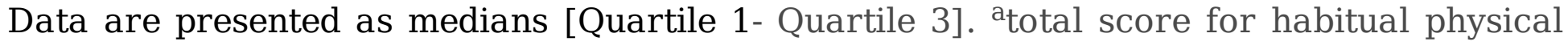
activity was obtained by summing physical exercise in leisure and leisure and locomotion activity scores. 
Table 3. Number of anti-hypertensive drugs used by participants

\begin{tabular}{lccc}
\hline Baecke's domains & Group 1 $(\mathrm{n}=14)$ & Group 2 $(\mathrm{n}=14)$ & $\boldsymbol{P}$ \\
\hline Total score & $2.0[1.0-3.0]$ & $2.0[1.0-2.3]$ & 0.77 \\
\hline
\end{tabular}

Data are presented as medians [Quartile 1-Quartile 3].

Table 4. Number of participants that used more than two antihypertensive drugs

\begin{tabular}{lccc}
\hline Number of antihypertensive drugs & Group 1 & Group 2 & $\boldsymbol{P}$ \\
\hline$\leq 2$ & $57.1(8)$ & $85.7(12)$ & 0.10 \\
$>2$ & $42.9(6)$ & $14.3(2)$ & \\
\hline
\end{tabular}

Data are presented as relative (absolute) frequencies. 